\title{
MAJORS OF FUNCTIONS
}

\author{
MIGUEL COUCEIRO AND ERKKO LEHTONEN
}

\begin{abstract}
We investigate the minor order of functions, focusing on upper covers and common upper bounds of pairs of functions. We show that two functions of arities $m$ and $n$ have a common upper bound if and only if they have a common lower bound, and if a common upper bound exists, then there is one of arity $m+n-1$. Moreover, we determine the possible essential arities of upper covers of functions.

Keywords: Function of several arguments, Boolean function, Essential arity, Minor order, Upper cover.
\end{abstract}

\section{INTRODUCTION}

This paper is a contribution to the understanding of the minor relation on the set $\mathcal{F}_{A B}$ of functions of several arguments from $A$ to $B$ : a function $f: A^{n} \rightarrow B$ is a minor of $g: A^{m} \rightarrow B$, or $g$ is a major of $f$, denoted $f \leq g$, if $f$ can be obtained from $g$ by identification of arguments, permutation of arguments, or introduction or deletion of inessential arguments. The minor relation constitutes a quasi-order on the set $\mathcal{F}_{A B}$, and, as usual, it induces an equivalence relation given by $f \equiv g$ if and only if $f \leq g$ and $g \leq f$, as well as a partial order on the quotient $\mathcal{F}_{A B} / \equiv$.

The structure of the minor partial order has been investigated in several studies, e.g., it was observed that the minor relation induces a partition of $\mathcal{F}_{A B}$ into blocks with no comparabilities in between (see Couceiro, Pouzet [5]). Each block comprises the functions sharing the same diagonal. By the diagonal of $f: A^{n} \rightarrow B$ we mean the function $\Delta f: A \rightarrow B$ given by $\Delta f(x)=f(x, \ldots, x)$.

The minor poset $\mathcal{F}_{A B} / \equiv$ has other nice properties. For example, all its principal down-sets are finite, and it is countable if $A$ and $B$ are finite. Moreover, if $A$ and $B$ are finite sets such that $|B| \geq \min (3,|A|)$, then the minor poset $\mathcal{F}_{A B} / \equiv$ is universal in the class of countable posets whose principal down-sets are finite (see Couceiro, Pouzet [5, Theorem 3], Lehtonen, Szendrei [6, Theorem 3.1]). Consequently, the descending chains are all finite, and this induces another partition of the minor poset into levels, each one of which is finite if $A$ and $B$ are finite.

Another interesting property is that in the case when $|A|=2$, the lower covers of $f: A^{n} \rightarrow B$ all have the same essential arity (see Bouaziz, Couceiro, Pouzet [2, Theorem 8]). This property is very particular to the case when $|A|=2$. Namely, Couceiro, Lehtonen, Waldhauser [4, Example 3.2] provided examples of functions with lower covers of different essential arities when $|A| \geq 5$. In the current paper, we provide further examples for all $|A| \geq 3$.

The very definition of minor provides a natural way of going downwards in the minor order, and it is perhaps due to this fact why most of the above-mentioned studies focused on the down-sets of the minor order. In contrast, the current paper is mainly concerned with going upwards in the minor order and the associated covering relation, in other words, with majors of functions. 
Our main goal is to describe the set of upper covers of a function $f: A^{n} \rightarrow B$. A full description still eludes us, but we take some steps towards it.

After setting up the necessary preliminaries, notation, and tools in Section 2, we will focus on three questions on majors of functions. Each one of the three subsequent sections begins with a question, which is then followed by theorems that provide an answer to it. More precisely, in Section 3, we study the following problem: given a function $f: A^{n} \rightarrow B$ and a positive integer $p$, what is the maximum number $\ell$ such that from every major of $f$ with $n+\ell$ essential arguments, we can get back to $f$, or to a minor of $f$, with $p$ identifications of arguments. It turns out that this maximum number is equal to $p$ for any function $f$.

In Section 4, we consider the question whether a common upper bound of two functions $f: A^{n} \rightarrow B$ and $g: A^{m} \rightarrow B$ exists. In fact, a common upper bound exists if and only if the two functions have the same diagonal. Furthermore, we show that if one exists, then there is one of essential arity $n+m-1$. The usefulness of this result is illustrated in Section 5 in a construction that shows that only Boolean functions have the property that their lower covers are of the same essential arity.

In Section 5, we describe the possible essential arities of the upper covers of a function $f: A^{n} \rightarrow B$. We conclude the paper with Section 6 , in which we discuss briefly the problem of enumerating the set of upper covers of a function.

\section{Preliminaries}

Let $\mathbb{N}$ be the set of nonnegative integers, and $\mathbb{N}_{+}=\mathbb{N} \backslash\{0\}$. For any $n \in \mathbb{N}$, we denote by $[n]$ the set $\{1, \ldots, n\}$ of the first $n$ positive integers. The set of all 2-element subsets of $[n]$ is denoted by $\left(\begin{array}{l}n \\ 2\end{array}\right)$.

This paper is a study of functions of several arguments, that is, mappings $f: A^{n} \rightarrow B$, where $A$ and $B$ are nonempty sets and $n \in \mathbb{N}_{+}$is called the arity of $f$. We will find it convenient to consider this notion in a slightly more general way, and we follow and adapt the formalism of Willard [7]. Namely, we will consider functions $f: A^{V} \rightarrow B$, where $A, B$, and $V$ are nonempty sets and $V$ is finite. The elements of $A^{V}$ are indexed families $\left(a_{i}\right)_{i \in V}$ of elements of $A$. If a linear order is specified, say $V=\{1, \ldots, n\}$ and $1<\cdots<n$, then the usual notations $\left(a_{1}, \ldots, a_{n}\right)$ and $f\left(a_{1}, \ldots, a_{n}\right)$ are used to denote tuples and values of $f$. We usually use bold letters to denote tuples and corresponding italic letters with subscripts to denote their components, e.g., $\mathbf{a}=\left(a_{1}, \ldots, a_{n}\right)$.

In what follows, the index set $V$ is often equal to $[n]$ for some $n \in \mathbb{N}$, and in this case we simply write $A^{n}$ for $A^{[n]}$, as usual, and the set $[n]$ is considered to be endowed with the natural linear order. In particular, our main results in the subsequent sections will be stated in this way. At the same time, in order to make our proofs more readable, we often take the set $V$ to be equal to a partition $\Pi$ of $[n]$, and in this case the $\Pi$-blocks are linearly ordered by their minimum elements.

Since a tuple $\mathbf{a} \in A^{T}$ is nothing but a map a: $T \rightarrow A$, the composition of a with any map $\sigma: S \rightarrow T$ is a well-defined tuple $\mathbf{a} \circ \sigma \in A^{S}$. We also write simply $\mathbf{a} \sigma$ instead of a $\circ \sigma$. For any map $\sigma: S \rightarrow T$, we define the map $\underline{\sigma}: A^{T} \rightarrow A^{S}$ by the rule $\underline{\sigma}(\mathbf{a})=\mathbf{a} \circ \sigma$ for all $\mathbf{a} \in A^{T}$, in other words, $\underline{\sigma}\left(\left(a_{t}\right)_{t \in T}\right)=\left(a_{\sigma(s)}\right)_{s \in S}$, for every $\left(a_{t}\right)_{t \in T} \in A^{T}$

Fact 1. Let $\sigma: S \rightarrow T$ and $\tau: T \rightarrow U$. Then $\underline{\sigma} \circ \underline{\tau}=\underline{\tau \circ \sigma}$. If $\sigma$ is a bijection, then $\underline{\sigma^{-1}}=(\underline{\sigma})^{-1}$. 
Recall that a partition of a set $S$ is a set $\Pi$ of pairwise disjoint nonempty subsets of $S$, the union of which equals $S$. The elements of a partition $\Pi$ are called blocks, or $\Pi$-blocks if we want to emphasize the partition that we are talking about. We refer to a partition with $\ell$ blocks as an $\ell$-partition. The $\Pi$-block containing the element $x \in S$ is denoted by $x / \Pi$. If $\Pi$ and $\Gamma$ are partitions of $S$ and every block of $\Pi$ is included in a block of $\Gamma$, then $\Pi$ is said to be a refinement of $\Gamma$, or $\Gamma$ is said to be a coarsening of $\Pi$, and we write $\Pi \sqsubseteq \Gamma$ to denote this fact. The set of all partitions of $S$ with the refinement relation $\sqsubseteq$ is a lattice, and we denote by $\Pi \vee \Gamma$ and $\Pi \wedge \Gamma$ the finest common coarsening and the coarsest common refinement of $\Pi$ and $\Gamma$, respectively. We order of the blocks of a partition $\Pi$ by their minimum elements: for $C, C^{\prime} \in \Pi$, we have $C \leq_{\Pi} C^{\prime}$ if and only if $\min C \leq \min C^{\prime}$.

Assume now that $V=[n]$ for some $n \in \mathbb{N}_{+}$. Let $\Pi$ be an $\ell$-partition of $[n]$. Let nat ${ }_{\Pi}:[n] \rightarrow \Pi$ be the natural surjection mapping each element $x$ of $[n]$ to the $\Pi$-block containing $x$. Let $\eta_{\Pi}:[\ell] \rightarrow \Pi$ be the order-isomorphism from $([\ell] ; \leq)$ to $\left(\Pi ; \leq_{\Pi}\right)$. Let $\delta_{\Pi}:[n] \rightarrow[\ell]$ be the mapping $\delta_{\Pi}=\left(\eta_{\Pi}\right)^{-1}$ onat ${ }_{\Pi}$. For any $f: A^{n} \rightarrow B$, define the functions $f_{\Pi}^{\prime}: A^{\Pi} \rightarrow B$ and $f_{\Pi}: A^{\ell} \rightarrow B$ as $f_{\Pi}^{\prime}=f \circ \underline{\text { nat }}$ and $f_{\Pi}=f \circ \underline{\delta_{\Pi}}$. By Fact 1, we have

$$
\left.f_{\Pi}=f \circ \underline{\delta_{\Pi}}=f \circ\left(\underline{\left(\eta_{\Pi}\right)^{-1} \circ \text { nat }_{\Pi}}\right)=f \circ \underline{\text { nat }_{\Pi}} \circ \underline{\left(\eta_{\Pi}\right)^{-1}}=f_{\Pi}^{\prime} \circ \underline{\left(\eta_{\Pi}\right.}\right)^{-1}
$$

and, consequently, $f_{\Pi}^{\prime}=f_{\Pi} \circ \underline{\eta_{\Pi}}$.

Remark 1 . The functions $f_{\Pi}$ and $f_{\Pi}^{\prime}$ are "essentially the same". The difference basically lies in relabeling the argument index set, the translation being provided by the bijection $\eta_{\Pi}:[\ell] \rightarrow \Pi$. We may thus switch between $f_{\Pi}$ and $f_{\Pi}^{\prime}$ at will and without difficulty, depending on which one of the two domains is more convenient to work with in each particular situation. We will, nevertheless, always explicitly indicate which one of the two functions, $f_{\Pi}$ or $f_{\Pi}^{\prime}$, we are considering in each case.

Let $I$ and $J$ be nonempty subsets of $V$. We denote by $\Pi_{I}$ the partition of $V$ whose only nontrivial block is $I$, and we let $\Pi_{I, J}:=\Pi_{I} \vee \Pi_{J}$. In order to simplify notation, we often write $I$ and $I, J$ instead of $\Pi_{I}$ and $\Pi_{I, J}$ in notations where symbols representing partitions occur as sub- or superscripts. For example, we write $f_{I}, f_{I}^{\prime}, \delta_{I}$ instead of the more formally correct $f_{\Pi_{I}}, f_{\Pi_{I}}^{\prime}, \delta_{\Pi_{I}}$.

Let $f: A^{V} \rightarrow B$. For any $i \in V$, we say that argument $i$ is essential in $f$, or $f$ depends on argument $i$, if there exist tuples $\mathbf{a}=\left(a_{j}\right)_{j \in V}$ and $\mathbf{b}=\left(b_{j}\right)_{j \in V}$ such that $a_{j}=b_{j}$ for all $j \in V \backslash\{i\}$ and $f(\mathbf{a}) \neq f(\mathbf{b})$. In this case, we say that $\mathbf{a}$ and $\mathbf{b}$ witness (or are witnesses of) the essentiality of argument $i$ in $f$. An argument that is not essential is inessential. We denote by Ess $f$ the set of essential arguments of $f$, i.e., Ess $f=\{i \in V$ : argument $i$ is essential in $f\}$. The cardinality of Ess $f$ is called the essential arity of $f$, or the number of essential arguments of $f$, and it is denoted by ess $f$.

A partial function of several arguments is a map $f: S \rightarrow B$, where $S \subseteq A^{V}$. Essential arguments can be defined for partial functions as above, but we must additionally require that witnesses of essentiality of arguments are members of the domain $S$ of $f$.

In order to illustrate the notions and notation introduced above, we provide a detailed proof of a simple - almost obvious - statement: If each member of a group of arguments is inessential in a function and we identify them into a single argument (possibly also performing some more identifications among other arguments), then this new argument will be inessential in the resulting minor. This statement could 
be formalized as follows: Given a map $f: A^{V} \rightarrow B$ and partitions $\Pi$ and $\Gamma$ of $V$, if $\Pi$ is a refinement of $\Gamma$, then for every $\Gamma$-block $I$ it holds that if the argument $J$ is inessential in $f_{\Pi}^{\prime}$ for every $\Pi$-block $J \subseteq I$, then $I$ is inessential in $f_{\Gamma}^{\prime}$. In Lemma 1 , we state and prove the contrapositive.

Lemma 1. Let $f: A^{V} \rightarrow B$. Let $\Pi$ and $\Gamma$ be partitions of $V$. If $\Pi \sqsubseteq \Gamma$, then for every $I \in \operatorname{Ess} f_{\Gamma}^{\prime}$, there exists $J \in \Pi$ such that $J \subseteq I$ and $J \in \operatorname{Ess} f_{\Pi}^{\prime}$.

Proof. Let $I \in \operatorname{Ess} f_{\Gamma}^{\prime}$, and let $J_{1}, \ldots, J_{p}$ be the distinct $\Pi$-blocks included in $I$. Let $\mathbf{a}=\left(a_{i}\right)_{i \in \Gamma}$ and $\mathbf{b}=\left(b_{i}\right)_{i \in \Gamma}$ be tuples witnessing the essentiality of argument $I$ in $f_{\Gamma}^{\prime}$, i.e., $a_{i}=b_{i}$ for all $i \neq I$ and $f_{\Gamma}^{\prime}(\mathbf{a}) \neq f_{\Gamma}^{\prime}(\mathbf{b})$. Define tuples $\mathbf{c}^{\ell}=\left(c_{i}^{\ell}\right)_{i \in \Pi}$ $(0 \leq \ell \leq p)$ as follows: $\mathbf{c}^{0}$ is the unique tuple in $A^{\Pi}$ satisfying $\underline{\operatorname{nat}_{\Pi}}\left(\mathbf{c}^{0}\right)=\underline{\operatorname{nat}_{\Gamma}}(\mathbf{a})$. For each $\ell \in\{1, \ldots, p\}, c_{i}^{\ell-1}=c_{i}^{\ell}$ for every $i \in \Pi \backslash\left\{J_{\ell}\right\}$, while $c_{J_{\ell}}^{\ell-1}=\overline{a_{I}}$ and $c_{J_{\ell}}^{\ell}=b_{I}$; in other words, $\mathbf{c}^{\ell}$ is obtained from $\mathbf{c}^{\ell-1}$ by changing the entry indexed by $J_{\ell}$ from $a_{I}$ to $b_{I}$. Consequently, $\mathbf{c}^{p}$ satisfies $\underline{\operatorname{nat}_{\Pi}}\left(\mathbf{c}^{p}\right)=\underline{\operatorname{nat}_{\Gamma}}(\mathbf{b})$.

Since

$$
\begin{aligned}
& f_{\Pi}^{\prime}\left(\mathbf{c}^{0}\right)=f\left(\underline{\operatorname{nat}_{\Pi}}\left(\mathbf{c}^{0}\right)\right)=f\left(\underline{\operatorname{nat}_{\Gamma}}(\mathbf{a})\right)=f_{\Gamma}^{\prime}(\mathbf{a}) \neq \\
& f_{\Gamma}^{\prime}(\mathbf{b})=f\left(\underline{\operatorname{nat}_{\Gamma}}(\mathbf{b})\right)=f\left(\underline{\operatorname{nat}_{\Pi}}\left(\mathbf{c}^{p}\right)\right)=f_{\Pi}^{\prime}\left(\mathbf{c}^{p}\right),
\end{aligned}
$$

there must exist an index $\ell \in\{1, \ldots, p\}$ such that $f_{\Pi}^{\prime}\left(\mathbf{c}^{\ell-1}\right) \neq f_{\Pi}^{\prime}\left(\mathbf{c}^{\ell}\right)$. Moreover, $\mathbf{c}^{\ell-1}$ and $\mathbf{c}^{\ell}$ only differ at position $J_{\ell}$, so they witness the essentiality of argument $J_{\ell}$ in $f_{\Pi}^{\prime}$.

Let $f: A^{n} \rightarrow B$ and $g: A^{m} \rightarrow B$. We say that $f$ is a minor of $g$, or that $g$ is a major of $f$, and we write $f \leq g$, if there exists a map $\sigma:[m] \rightarrow[n]$ such that $f=g \circ \underline{\sigma}$, that is, $f(\mathbf{a})=g(\mathbf{a} \sigma)$ for all $\mathbf{a} \in A^{n}$. If $f \leq g$ and $g \leq f$, then we say that $f$ and $g$ are equivalent and write $f \equiv g$. As usual, we write $f<g$ to denote the fact that $f \leq g$ but $g \not \leq f$; in this case we say that $f$ is a proper minor of $g$. It is not difficult to verify that every minor of $f$ is equivalent to a function of the form $f_{\Pi}$ for some partition $\Pi$ of $[n]$.

An important special case of the minors of $f$ are the functions of the form $f_{I}$ for some $I \in\left(\begin{array}{l}n \\ 2\end{array}\right)$. (Recall that $f_{I}$ means $f_{\Pi_{I}}$, where $\Pi_{I}$ is the partition of $[n]$ whose only nontrivial block is $I$.) Such functions are referred to as identification minors of $f$. It is easy to write down explicitly a defining expression for the identification minors of $f$. Indeed, for any $I \in\left(\begin{array}{l}n \\ 2\end{array}\right)$, the function $f_{I}: A^{n-1} \rightarrow B$ is given by the rule $f_{I}=f \circ \underline{\delta_{I}}$, where $\delta_{I}:[n] \rightarrow[n-1]$ is the map

$$
\delta_{I}(i)= \begin{cases}i, & \text { if } i<\max I, \\ \min I, & \text { if } i=\max I \\ i-1, & \text { if } i>\max I\end{cases}
$$

In other words, if $I=\{i, j\}$ with $i<j$, then

$$
f_{I}\left(a_{1}, \ldots, a_{n-1}\right)=f\left(a_{1}, \ldots, a_{j-1}, a_{i}, a_{j}, \ldots, a_{n-1}\right),
$$

for all $a_{1}, \ldots, a_{n-1} \in A$. Note that $a_{i}$ occurs twice on the right side of the above equality: both at the $i$-th and at the $j$-th positions.

In the remainder of this paper, we assume that the sets $A$ and $B$ have at least two elements. For, if $|A|=1$ or $|B|=1$, then every function $f: A^{n} \rightarrow B$ is constant and ess $f=0$. 


\section{FROM HOW HIGH CAN WE JUMP BACK?}

Question 1. Let $f: A^{n} \rightarrow B$, and assume that $f$ depends on all of its arguments. For a fixed parameter $p \geq 1$, what is the maximum number $\ell$ such that for every major $g: A^{n+\ell} \rightarrow B$ of $f$ that depends on all of its arguments, there exists an $(n+\ell-p)$-partition $\Pi$ of $[n+\ell]$ such that $g_{\Pi} \leq f$ ? In particular (when $\left.p=1\right)$, what is the maximum number $\ell$ such that for every major $g: A^{n+\ell} \rightarrow B$ of $f$ that depends on all of its arguments, there exists $I \in\left(\begin{array}{c}n \\ 2\end{array}\right)$ such that $g_{I} \leq f$ ?

It is easy to see that the maximum number $\ell$ is at least $p$ : any identification of essential arguments decreases the essential arity, and since $g$ is a major of $f$, there must exist a partition $\Pi$ with at least $n$ blocks such that $g_{\Pi} \equiv f$. Proposition 2 shows that the maximum number is actually equal to $p$, for every function $f$.

The diagonal function of $f: A^{n} \rightarrow B$ is the function $\Delta f: A \rightarrow B$ given by the rule $\Delta f(a)=f(a, \ldots, a)$ for all $a \in A$. The function $\Delta f$ is a minor of $f$; in fact, $\Delta f=f_{\Pi}$, where $\Pi=\{[n]\}$.

Proposition 2. Let $f: A^{n} \rightarrow B$, and let $\ell \geq 1$. Then there exist a function $g: A^{n+\ell} \rightarrow B$ with ess $g=n+\ell$ and an n-partition $\Pi$ of $[n+\ell]$ such that $g_{\Pi}=f$, but there is no partition $\Gamma$ of $[n+\ell]$ with at least $n+1$ blocks such that $g_{\Gamma} \leq f$.

Proof. Fix elements $\alpha, \beta \in A$ with $\alpha \neq \beta$, and let $\phi: \mathcal{P}(A) \rightarrow B$ be a map that satisfies the following conditions: $\Delta f(\alpha) \neq \phi(\{\alpha\}), \phi(\{\alpha\}) \neq \phi(\{\alpha, \beta\})$, $f(\beta, \beta, \ldots, \beta, \alpha) \neq \phi(\{\beta\})$. It is clear that such a function $\phi$ exists. Define $g: A^{n+\ell} \rightarrow B$ by the rule

$$
g\left(a_{1}, \ldots, a_{n+\ell}\right)= \begin{cases}f\left(a_{1}, \ldots, a_{n}\right), & \text { if } a_{n}=a_{n+1}=\cdots=a_{n+\ell}, \\ \phi\left(\left\{a_{1}, \ldots, a_{n}\right\}\right), & \text { otherwise }\end{cases}
$$

It is clear that $f=g_{\Pi}$ for the $n$-partition $\Pi=\{\{1\},\{2\}, \ldots,\{n-1\},\{n, \ldots, n+\ell\}\}$. We are going to show that for every $p$-partition $\Gamma$ of $[n+\ell]$ with $p \geq n+1$, the function $g_{\Gamma}^{\prime}$ depends on all of its arguments. Then it is not possible that $g_{\Gamma} \leq f$, because ess $g_{\Gamma}=p \geq n+1>$ ess $f$. Moreover, ess $g=n+\ell$.

Let $\Gamma$ be a $p$-partition of $[n+\ell]$ with $p \geq n+1$. Then there exists a $\Gamma$-block $Q$ that is a subset of $\{n+1, \ldots, n+\ell\}$. (Otherwise every $\Gamma$-block would contain an element of the set $\{1, \ldots, n\}$, and there would be at most $n$ distinct $\Gamma$-blocks, a contradiction.) We will show that $P \in \operatorname{Ess} g_{\Gamma}^{\prime}$ for every $P \in \Gamma$. We consider four different cases that exhaust all possibilities.

Case 1: $P \subseteq\{n+1, \ldots, n+\ell\}$. Let $\mathbf{u}, \mathbf{u}^{\prime} \in A^{\Gamma}$ be tuples such that $u_{P}=\alpha$, $u_{P}^{\prime}=\beta$, and $u_{T}=u_{T}^{\prime}=\alpha$ for all $T \in \Gamma \backslash\{P\}$. Then $g_{\Gamma}^{\prime}(\mathbf{u})=\Delta f(\alpha) \neq \phi(\{\alpha\})=$ $g_{\Gamma}^{\prime}\left(\mathbf{u}^{\prime}\right)$, so $P \in \operatorname{Ess} g_{\Gamma}^{\prime}$.

Case 2: $P \nsubseteq\{n+1, \ldots, n+\ell\},\{n, \ldots, n+\ell\} \nsubseteq P \cup Q$, and $\{1, \ldots, n\} \nsubseteq P$. Let $\mathbf{u}, \mathbf{u}^{\prime} \in A^{\Gamma}$ be tuples such that $u_{P}=\alpha, u_{P}^{\prime}=\beta, u_{Q}=u_{Q}^{\prime}=\beta$, and $u_{T}=u_{T}^{\prime}=\alpha$ for all $T \in \Gamma \backslash\{P, Q\}$. Then $g_{\Gamma}^{\prime}(\mathbf{u})=\phi(\{\alpha\}) \neq \phi(\{\alpha, \beta\})=g_{\Gamma}^{\prime}\left(\mathbf{u}^{\prime}\right)$, so $P \in \operatorname{Ess} g_{\Gamma}^{\prime}$.

Case 3: $P \nsubseteq\{n+1, \ldots, n+\ell\},\{n, \ldots, n+\ell\} \nsubseteq P \cup Q$, and $\{1, \ldots, n\} \subseteq P$. We need to consider two cases according to whether or not $\phi(\{\alpha\})$ and $\phi(\{\beta\})$ are equal. If $\phi(\{\alpha\})=\phi(\{\beta\})$, then let $\mathbf{u}, \mathbf{u}^{\prime} \in A^{\Gamma}$ be tuples such that $u_{P}=\alpha, u_{P}^{\prime}=\beta$, $u_{Q}=u_{Q}^{\prime}=\alpha$, and $u_{T}=u_{T}^{\prime}=\alpha$ for all $T \in \Gamma \backslash\{P, Q\}$. Then $g_{\Gamma}^{\prime}(\mathbf{u})=\Delta f(\alpha) \neq$ $\phi(\{\alpha\})=\phi(\{\beta\})=g_{\Gamma}^{\prime}\left(\mathbf{u}^{\prime}\right)$, so $P \in \operatorname{Ess} g_{\Gamma}^{\prime}$. If $\phi(\{\alpha\}) \neq \phi(\{\beta\})$, then let $\mathbf{u}, \mathbf{u}^{\prime} \in A^{\Gamma}$ be tuples such that $u_{P}=\alpha, u_{P}^{\prime}=\beta, u_{Q}=u_{Q}^{\prime}=\beta$, and $u_{T}=u_{T}^{\prime}=\alpha$ for all $T \in \Gamma \backslash\{P, Q\}$. Then $g_{\Gamma}^{\prime}(\mathbf{u})=\phi(\{\alpha\}) \neq \phi(\{\beta\})=g_{\Gamma}^{\prime}\left(\mathbf{u}^{\prime}\right)$, so $P \in \operatorname{Ess} g_{\Gamma}^{\prime}$. 
Case 4: $P \nsubseteq\{n+1, \ldots, n+\ell\}$ and $\{n, \ldots, n+\ell\} \subseteq P \cup Q$. Since $p \geq n+1$, this means in fact that $p=n+1, P=\{n, \ldots, n+\ell\} \backslash Q$ and the singletons $\{i\}, i \in[n-1]$ are $\Gamma$-blocks. Let $\mathbf{u}, \mathbf{u}^{\prime} \in A^{\Gamma}$ be tuples satisfying $u_{\{i\}}=u_{\{i\}}^{\prime}=\beta$ for all $i \in[n-1]$, $u_{P}=\alpha, u_{P}^{\prime}=\beta, u_{Q}=u_{Q}^{\prime}=\alpha$. Then $g_{\Gamma}^{\prime}(\mathbf{u})=f(\beta, \ldots, \beta, \alpha) \neq \phi(\{\beta\})=g_{\Gamma}^{\prime}\left(\mathbf{u}^{\prime}\right)$, so $P \in \operatorname{Ess} g_{\Gamma}^{\prime}$.

\section{Common upper Bounds}

Question 2. Under which conditions do two functions have a common upper bound in the minor order? What is the minimum essential arity of the common upper bounds, if one exists?

Recall that two functions $f: A^{m} \rightarrow B, g: A^{n} \rightarrow B$ have a common lower bound in the minor order if and only if $\Delta f=\Delta g$. In this section, we show that whenever $f$ and $g$ have a common lower bound, then they also have a common upper bound. Moreover, if a common upper bound of $f$ and $g$ exists, then there exists one of arity $m+n-1$. It is not, however, in general guaranteed that upper bounds of smaller arity exist, as illustrated by Example 1.

Proposition 3. Let $f: A^{m} \rightarrow B, g: A^{n} \rightarrow B$, and assume that $\Delta f=\Delta g$. Then there exists a function $h: A^{m+n-1} \rightarrow B$ such that $f$ and $g$ are minors of $h$.

Proof. Without loss of generality, assume that $m \leq n$. Let $h: A^{m+n-1} \rightarrow B$ be any function that satisfies

$$
h\left(a_{1}, \ldots a_{m+n-1}\right)= \begin{cases}f\left(a_{1}, \ldots, a_{m}\right), & \text { if } a_{m}=\cdots=a_{m+n-1} \\ g\left(a_{m}, \ldots, a_{m+n-1}\right), & \text { if } a_{1}=\cdots=a_{m}\end{cases}
$$

Note that such a function $h$ exists, because we are assuming that $\Delta f=\Delta g$. It is clear that $f=h_{\Pi}$ and $g=h_{\Gamma}$, where

$$
\begin{aligned}
\Pi & =\{\{1\}, \ldots,\{m-1\},\{m, \ldots, m+n-1\}\}, \\
\Gamma & =\{\{1, \ldots, m\},\{m+1\}, \ldots,\{m+n-1\}\} .
\end{aligned}
$$

Lemma 4. Let $n \geq 1$. Let $\Pi$ and $\Gamma$ be partitions of $[2 n]$ with at least $n+1$ blocks. Then $\Pi \vee \Gamma$ has at least two blocks.

Proof. It is well known that lattices of partitions of finite sets are semimodular and hence satisfy the Jordan-Hölder chain condition: any two maximal chains have the same length. Denoting by $h(\Pi)$ the height of a partition $\Pi$ on a set $A$ (i.e., the common length of the maximal chains connecting $\Pi$ to the bottom of the partition lattice), we have $h(\Pi)+h(\Gamma) \geq h(\Pi \vee \Gamma)+h(\Pi \wedge \Gamma)$, for all partitions $\Pi$, $\Gamma$. Since $h(\Pi)=|A|-|\Pi|$, this implies $|\Pi|+|\Gamma| \leq|\Pi \vee \Gamma|+|\Pi \wedge \Gamma|$. Thus, $|\Pi \wedge \Gamma| \geq|\Pi|+|\Gamma|-|\Pi \vee \Gamma| \geq(n+1)+(n+1)-2 n=2$.

As it will become clear from the following example, we cannot improve the arity $m+n-1$ of the common upper bound of $f$ and $g$ in Proposition 3 . 
Example 1. Assume that 0 and 1 are distinct elements of $B$. Define the functions $f, g: A^{n} \rightarrow B$ as

$$
\begin{aligned}
& f\left(a_{1}, \ldots, a_{n}\right)= \begin{cases}0, & \text { if } a_{1}=\cdots=a_{n}=0, \\
1, & \text { otherwise }\end{cases} \\
& g\left(a_{1}, \ldots, a_{n}\right)= \begin{cases}1, & \text { if } a_{1}=\cdots=a_{n} \neq 0, \\
0, & \text { otherwise }\end{cases}
\end{aligned}
$$

Obviously $\Delta f=\Delta g$, so by Proposition 3, there exists $h: A^{2 n-1} \rightarrow B$ such that $f \leq h$ and $g \leq h$.

We claim that there does not exist any $h: A^{2 n-2} \rightarrow B$ such that $f \leq h$ and $g \leq h$. Suppose, to the contrary, that there is such a function $h$. Then there exist $n$-partitions $\Pi$ and $\Gamma$ of $[2 n-2]$ such that $h_{\Pi}=f$ and $h_{\Gamma}=g$. (Note that $f$ and $g$ are totally symmetric, so we can take equality and not just equivalence here.) By Lemma 4 , the partition $\Pi \vee \Gamma$ has at least two blocks. Let $B$ be a block of $\Pi \vee \Gamma$, let $\alpha$ and $\beta$ be distinct elements of $A$, and let $\mathbf{u} \in A^{2 n-2}$ be the tuple satisfying $u_{i}=\alpha$ for $i \in B$, and $u_{i}=\beta$ for $i \in[2 n-2] \backslash B$. Then $\mathbf{u}=\mathbf{v} \delta_{\Pi}=\mathbf{w} \delta_{\Gamma}$ for some $\mathbf{v}, \mathbf{w} \in A^{n}$ and $\mathbf{v}$ and $\mathbf{w}$ are not constant tuples. Therefore,

$$
1=f(\mathbf{v})=h_{\Pi}(\mathbf{v})=h\left(\mathbf{v} \delta_{\Pi}\right)=h(\mathbf{u})=h\left(\mathbf{w} \delta_{\Gamma}\right)=h_{\Gamma}(\mathbf{w})=g(\mathbf{w})=0,
$$

a contradiction.

\section{UPPER COVERS}

We say that $f$ is a lower cover of $g$, or that $g$ is an upper cover of $f$, if $f<g$ in the minor order and there is no $h$ such that $f<h<g$. Denote by $C_{f}$ the set of upper covers of $f$.

Question 3. Given a function $f: A^{n} \rightarrow B$ with ess $f=n$, what are the possible essential arities of the upper covers of $f$ ? In other words, describe the set $\{\operatorname{ess} g$ : $\left.g \in C_{f}\right\}$. In particular, what is $\max \left\{\operatorname{ess} g: g \in C_{f}\right\}$ ?

To give an answer to this question, we will make use of the mapping oddsupp: $\bigcup_{n \geq 1} A^{n} \rightarrow \mathcal{P}(A)$ defined by Berman and Kisielewicz [1] as follows:

$$
\operatorname{oddsupp}\left(a_{1}, \ldots, a_{n}\right)=\left\{a \in A:\left|\left\{i \in[n]: a_{i}=a\right\}\right| \text { is odd }\right\} \text {. }
$$

A function $f: A^{n} \rightarrow B$ is then said to be determined by oddsupp if $f=f^{*} \circ$ oddsupp $\left.\right|_{A^{n}}$ for some $f^{*}: \mathcal{P}(A) \rightarrow B$.

Theorem 5. Assume that $f: A^{n} \rightarrow B$ is a function that depends on all of its arguments. Let $\ell \in \mathbb{N}_{+}$.

(i) If $\ell \geq \max (|A|, 3)$, then $f$ does not have upper covers of essential arity $n+\ell$.

(ii) If $1 \leq \ell<|A|$, then there exists an upper cover of $f$ of essential arity $n+\ell$.

(iii) If $|A|=2$, then $f$ has an upper cover of essential arity $n+2$ if and only if $f$ is determined by oddsupp.

Note that Theorem 5(ii) asserts that if $A$ is infinite, then $f$ has upper covers of essential arity $n+\ell$ for every $\ell \in \mathbb{N}_{+}$.

The remainder of this section constitutes the proof of Theorem 5. First we need to introduce several concepts and prove some auxiliary results.

The following result was proved by Bouaziz, Couceiro, Pouzet [2, Theorem 8]. It was originally stated for Boolean functions but the provided proof is actually 
valid in the more general setting of functions $f: A^{n} \rightarrow B$, where $|A|=2$ and the codomain $B$ is arbitrary.

Theorem 6. Assume that $|A|=2$ and $f: A^{n} \rightarrow B$ depends on all of its arguments. Then all lower covers of $f$ have the same essential arity that is either $n-1$ or $n-2$. In the latter case $f$ has a unique lower cover.

Note, however, that this result is very particular to the case when $|A|=2$. The statement would no longer be true if we removed the condition $|A|=2$. This was illustrated by Couceiro, Lehtonen, Waldhauser [4, Example 3.2] with a 6-ary operation on a 5-element set that has lower covers of essential arity 4 and 5 . This example can be easily adapted to functions over larger sets $A$. However, that example left open the question whether there exist functions $f: A^{n} \rightarrow B$ with lower covers of different essential arities in the case when $|A| \in\{3,4\}$. The following example provides a positive answer.

Write $A_{\neq}^{n}:=\left\{\left(a_{1}, \ldots, a_{n}\right) \in A^{n}: a_{i}=a_{j} \Longrightarrow i=j\right\}$.

Example 2. Assume that $|A| \geq 3$ and that 0 and 1 are distinct elements of $B$. Let $f: A^{2} \rightarrow B$ and $g: A^{3} \rightarrow B$ be functions given by the rules

$$
\begin{aligned}
f\left(x_{1}, x_{2}\right) & = \begin{cases}0, & \text { if } x_{1}=x_{2}, \\
1, & \text { if } x_{1} \neq x_{2},\end{cases} \\
g\left(x_{1}, x_{2}, x_{3}\right) & = \begin{cases}0, & \text { if }\left(x_{1}, x_{2}, x_{3}\right) \notin A_{\neq}^{3}, \\
1, & \text { if }\left(x_{1}, x_{2}, x_{3}\right) \in A_{\neq}^{3} .\end{cases}
\end{aligned}
$$

We apply the construction given in the proof of Proposition 3 to produce a common upper bound of $f$ and $g$, namely, the function $h: A^{4} \rightarrow B$ given by

$$
h\left(x_{1}, x_{2}, x_{3}, x_{4}\right)= \begin{cases}f\left(x_{1}, x_{3}\right), & \text { if } x_{1}=x_{2} \text { and } x_{3}=x_{4}, \\ g\left(x_{1}, x_{3}, x_{4}\right), & \text { if } x_{2}=x_{3}, \\ 1, & \text { otherwise. }\end{cases}
$$

It is clear from Proposition 3 that $f$ and $g$ are minors of $h$. Moreover, $g$ cannot be a minor of $f$, because ess $f<\operatorname{ess} g$, and $f$ cannot be a minor of $g$, because any identification of arguments in $g$ yields a constant function. It remains to show that $f$ and $g$ are lower covers of $h$. Clearly, we have $h_{I}=g$ for $I=\{2,3\}$. Moreover, it is not difficult to verify that whenever $I \in\{\{1,2\},\{1,3\}\}$, the equality $h_{I}\left(x_{1}, x_{2}, x_{3}\right)=$ $f\left(x_{1}, x_{2}\right)$ holds for all $x_{1}, x_{2}, x_{3} \in A$, and whenever $I \in\{\{1,4\},\{2,4\},\{3,4\}\}$, the equality $h_{I}\left(x_{1}, x_{2}, x_{3}\right)=f\left(x_{2}, x_{3}\right)$ holds for all $x_{1}, x_{2}, x_{3} \in A$. Consequently, $h_{I} \equiv f$ for all $I \neq\{2,3\}$. This shows that $f$ and $g$ are indeed lower covers of $h$ with different essential arities.

The arity gap of a function $f: A^{n} \rightarrow B$ is the minimum difference between the essential arity of $f$ and that of its proper minors: $\operatorname{gap} f=\min _{g<f}(\operatorname{ess} f-\operatorname{ess} g)$. If $f$ depends on all of its arguments, this is the same as $\min _{I \in\left(\begin{array}{c}n \\ 2\end{array}\right)}\left(\operatorname{ess} f-\operatorname{ess} f_{I}\right)$. For further information on the arity gap, see Couceiro, Lehtonen [3]. The following result on the arity gap is sufficient for our needs.

Theorem 7 ([3, Theorem 13]). For a pseudo-Boolean function $f:\{0,1\}^{n} \rightarrow B$, $n \geq 2$, which depends on all of its variables, gap $f=2$ if and only if $f$ satisfies one of the following conditions:

- $n=2$ and $f$ is a nonconstant function satisfying $f(0,0)=f(1,1)$, 
- $f=g \circ h$, where $g:\{0,1\} \rightarrow B$ is injective and $h:\{0,1\}^{n} \rightarrow\{0,1\}$ is a Boolean function with gap $h=2$, as listed below:

$-x_{1}+x_{2}+\cdots+x_{m}+c(2 \leq m \leq n)$,

$-x_{1} x_{2}+x_{1}+c$,

$-x_{1} x_{2}+x_{1} x_{3}+x_{2} x_{3}+c$

$-x_{1} x_{2}+x_{1} x_{3}+x_{2} x_{3}+x_{1}+x_{2}+c$,

where addition and multiplication are done modulo 2 and $c \in\{0,1\}$.

Otherwise gap $f=1$.

Proposition 8. Assume that $|A|=2$ and $f: A^{n} \rightarrow B$ depends on all of its arguments. Then $f$ has an upper cover with $n+2$ essential arguments if and only if $f$ is determined by oddsupp.

Proof. If $f=f^{*}$ o oddsupp $\left.\right|_{A^{n}}$, then the function $g=f^{*} \circ$ oddsupp $\left.\right|_{A^{n+2}}$ has a unique identification minor, namely $f$. Therefore $g$ is an upper cover of $f$ with $\operatorname{ess} g=\operatorname{ess} f+2$.

If $f$ has an upper cover $g: A^{n+2} \rightarrow B$ that depends on all of its arguments, then it follows from Theorem 6 that gap $g=2$. Moreover, by Theorem 7, either $g$ is at most ternary or $g$ is determined by oddsupp. It follows that $f$ is determined by oddsupp. Namely, if $g$ is at most ternary, then $f$ is unary and the claim is true, because all unary functions are determined by oddsupp. The claim holds also in the case when $g$ is determined by oddsupp, because then all minors of $g$ are determined by oddsupp.

Proposition 9. Let $n, \ell \in \mathbb{N}_{+}, f: A^{n} \rightarrow B$ and $g: A^{\ell} \rightarrow B$, and assume that ess $f=n$ and $\operatorname{ess} g=\ell$. If $\ell \geq n+\max (|A|, 3)$, then $g$ is not an upper cover of $f$.

Observe that in Proposition 9, it is necessary to assume that $\ell \geq n+3$, because in the case of Boolean functions, i.e., $|A|=2$, any linear function of arity $n$ has an upper cover of arity $n+2$ and would thus falsify the statement.

Proof. Suppose, to the contrary, that $g$ is an upper cover of $f$. Then there exists $L \in$ $\left(\begin{array}{l}\ell \\ 2\end{array}\right)$ such that $f \equiv g_{L}$. Permuting the arguments of $g$ in a suitable way if necessary, we may assume without loss of generality that $g_{L}\left(x_{1}, \ldots, x_{\ell-1}\right)=f\left(x_{1}, \ldots, x_{n}\right)$. Thus Ess $g_{L}^{\prime}=\left\{i / \Pi_{L}: 1 \leq i \leq n\right\}$. Furthermore, we may assume that if $L \in \operatorname{Ess} g_{L}^{\prime}$ then $L=\{n, n+1\}$, and if $L \notin \operatorname{Ess} g_{L}^{\prime}$, then $L=\{\ell-1, \ell\}$. Write $E_{L}:=\bigcup \operatorname{Ess} g_{L}^{\prime}$. We thus have $E_{L}=\{1, \ldots, n+1\}$ if $L \in \operatorname{Ess} g_{L}^{\prime}$, and $E_{L}=\{1, \ldots, n\}$ if $L \notin \operatorname{Ess} g_{L}^{\prime}$.

Observe that for every $I \in\left(\begin{array}{c}\ell \\ 2\end{array}\right)$ such that $I \nsubseteq E_{L}$ and $I \neq L$, it holds that $g_{L, I} \equiv$ $f$. In fact, $g_{L, I}\left(x_{1}, \ldots x_{\ell-2}\right)=f\left(x_{1}, \ldots, x_{n}\right)$. Moreover, since $f \equiv g_{L, I} \leq g_{I}<g$ and since $g$ is an upper cover of $f$, we must have that $g_{I} \equiv f$.

Furthermore, we may assume that $L \in \operatorname{Ess} g_{L}^{\prime}$. For, if $L \notin \operatorname{Ess} g_{L}^{\prime}$, then let $L^{\prime}$ be any couple in $\left(\begin{array}{l}\ell \\ 2\end{array}\right)$ such that $L^{\prime} \nsubseteq E_{L}, L^{\prime} \cap E_{L} \neq \emptyset$, and $L \cap L^{\prime}=\emptyset$. Such a couple $L^{\prime}$ exists, because we are assuming that $n \geq 1$ and $\ell \geq n+3$. Then Ess $g_{L, L^{\prime}}^{\prime}=\operatorname{Ess} g_{L}^{\prime} \backslash\left\{E_{L} \cap L^{\prime}\right\} \cup\left\{L^{\prime}\right\}$; thus $L^{\prime} \in \operatorname{Ess} g_{L, L^{\prime}}^{\prime}$. Lemma 1 then asserts that $L^{\prime} \in \operatorname{Ess} g_{L^{\prime}}^{\prime}$, and we have $g_{L^{\prime}} \equiv f$. Therefore we may consider $L^{\prime}$ instead of $L$.

Thus we have $L=\{n, n+1\}$, Ess $g_{L}^{\prime}=\{\{1\}, \ldots,\{n-1\},\{n, n+1\}\}$, and $E_{L}=\{1, \ldots, n+1\}$. Let $I=\{i, j\} \in\left(\begin{array}{l}\ell \\ 2\end{array}\right)(i<j)$ with $I \nsubseteq E_{L}$. Let us determine Ess $g_{I}^{\prime}$ in terms of Ess $g_{L}^{\prime}$, by applying Lemma 1 and taking into account that $g_{I} \equiv f$ and hence ess $g_{I}^{\prime}=n$. 
- If $I \cap E_{L}=\emptyset$, then Ess $g_{L, I}^{\prime}=\operatorname{Ess} g_{L}^{\prime}$, because the arguments $i / \Pi_{L}$ for $i \in I$ are inessential in $g_{L}^{\prime}$, and Ess $g_{I}^{\prime}=\left(\operatorname{Ess} g_{L}^{\prime} \backslash\{L\}\right) \cup\left\{\left\{\ell_{I}\right\}\right\}$ for some $\ell_{I} \in L$.

- If $i \in E_{L} \backslash L$ and $j \notin E_{L}$, then Ess $g_{L, I}^{\prime}=\left(\operatorname{Ess} g_{L}^{\prime} \backslash\{\{i\}\}\right) \cup\{I\}$, and $\operatorname{Ess} g_{I}^{\prime}=\left(\operatorname{Ess} g_{L, I}^{\prime} \backslash\{L\}\right) \cup\left\{\left\{\ell_{I}\right\}\right\}=\left(\operatorname{Ess} g_{L}^{\prime} \backslash\{\{i\}, L\}\right) \cup\left\{I,\left\{\ell_{I}\right\}\right\}$ for some $\ell_{I} \in L$.

- If $i \in L$ and $j \notin E_{L}$, then $\operatorname{Ess} g_{L, I}^{\prime}=\left(\operatorname{Ess} g_{L}^{\prime} \backslash\{L\}\right) \cup\{L \cup I\}$, and $\operatorname{Ess} g_{I}^{\prime}=$ $\left(\operatorname{Ess}_{L}^{\prime} \backslash\{L\}\right) \cup\left\{\ell_{I} / \Pi_{I}\right\}$ for some $\ell_{I} \in L$. Note that in this case $\ell_{I} / \Pi_{I}$ equals either $I$ or $L \backslash I$.

We can summarize the above as follows: for each $I \in\left(\begin{array}{l}\ell \\ 2\end{array}\right)$ with $I \nsubseteq E_{L}$, there exists a unique element $\ell_{I} \in L$ such that $\operatorname{Ess} g_{I}^{\prime}=\left\{i / \Pi_{I}: i \in E_{L} \backslash L\right\} \cup\left\{\ell_{I} / \Pi_{I}\right\}$. Recall that $i / \Pi_{I}$ denotes the block containing $i$ in the partition $\Pi_{I}$, in which $I$ is the only nontrivial block.

Claim 1. For any $I=\left\{i_{1}, i_{2}\right\} \in\left(\begin{array}{l}\ell \\ 2\end{array}\right)\left(i_{1}<i_{2}\right)$ with $I \nsubseteq E_{L}$, it holds that $g_{I}\left(a_{1}, \ldots, a_{\ell-1}\right)=f\left(a_{1}, \ldots, a_{n-1}, a_{\ell_{I}}\right)$.

Proof. In order to see this, recall two facts that we have established above: Ess $g_{I}^{\prime}=$ $\left\{i / \Pi_{I}: i \in E_{L} \backslash L\right\} \cup\left\{\ell_{I} / \Pi_{I}\right\}$ and $g_{I} \equiv f$. It follows from these facts that there exists a permutation $\sigma_{I}$ of $[n]$ such that $g_{I}\left(a_{1}, \ldots, a_{\ell-1}\right)=f\left(\left(a_{1}, \ldots, a_{n-1}, a_{\ell_{I}}\right) \sigma_{I}\right)$. We also have $g_{L}\left(a_{1}, \ldots, a_{\ell-1}\right)=f\left(a_{1}, \ldots, a_{n}\right)$. Now, let $\mathbf{a}=\left(a_{1}, \ldots, a_{n}\right) \in A^{n}$ be arbitrary, and let $\mathbf{b}=\left(b_{1}, \ldots, b_{\ell}\right) \in A^{\ell}$ be any tuple such that $b_{i}=a_{i}$ for all $i \in[n]$, $b_{n+1}=b_{n}$ and $b_{i_{1}}=b_{i_{2}}$. It is easy to see that such a tuple $\mathbf{b}$ exists. Then there exist tuples $\mathbf{b}^{\prime}, \mathbf{b}^{\prime \prime} \in A^{\ell-1}$ such that $\mathbf{b}^{\prime} \delta_{I}=\mathbf{b}=\mathbf{b}^{\prime \prime} \delta_{L}$; furthermore, $b_{i}=b_{i}^{\prime}=b_{i}^{\prime \prime}$ for all $i \in[n]$. We have

$$
g(\mathbf{b})=g\left(\mathbf{b}^{\prime \prime} \delta_{L}\right)=g_{L}\left(\mathbf{b}^{\prime \prime}\right)=f(\mathbf{a}) .
$$

Moreover, since $b_{n}=b_{n+1}$ and $\ell_{I} \in\{n, n+1\}$, we also have

$$
g(\mathbf{b})=g\left(\mathbf{b}^{\prime} \delta_{I}\right)=g_{I}\left(\mathbf{b}^{\prime}\right)=f\left(\left(b_{1}, \ldots, b_{n-1}, b_{\ell_{I}}\right) \sigma_{I}\right)=f\left(\mathbf{a} \sigma_{I}\right) .
$$

Putting the above two equalities together, we get that the equality $f(\mathbf{a})=f\left(\mathbf{a} \sigma_{I}\right)$ holds for all $\mathbf{a} \in A^{n}$. Consequently,

$$
g_{I}\left(a_{1}, \ldots, a_{\ell-1}\right)=f\left(\left(a_{1}, \ldots, a_{n-1}, a_{\ell_{I}}\right) \sigma_{I}\right)=f\left(a_{1}, \ldots, a_{n-1}, a_{\ell_{I}}\right),
$$

as claimed.

Claim 2. $\ell_{I}=\ell_{J}$ for all $I, J \in\left(\begin{array}{l}\ell \\ 2\end{array}\right)$ with $I, J \nsubseteq E_{L}$.

Proof. Suppose, to the contrary, that $\ell_{I} \neq \ell_{J}$ for some $I=\left\{i_{1}, i_{2}\right\}$ and $J=\left\{j_{1}, j_{2}\right\}$ with $i_{2}, j_{2} \notin \operatorname{Ess} g_{L}$. We may assume that if $i_{1}$ and $j_{1}$ are both in $E_{L}$, then $i_{2} \neq j_{2}$. For, if $i_{1}, j_{1} \in E_{L}$ and $i_{2}=j_{2}$, then, since $\ell \geq n+3$, and $\left|E_{L}\right|=n+1$, there exists an element $k_{2} \in[\ell] \backslash\left(E_{L} \cup\left\{i_{2}\right\}\right)$. Let $K=\left\{i_{2}, k_{2}\right\}$. Then either $\ell_{K} \neq \ell_{I}$ or $\ell_{K} \neq \ell_{J}$. Therefore, instead of $I$ and $J$, we may consider the couples $K$ and the one among $I$ and $J$ for which the inequality holds.

Assume, without loss of generality, that $\ell_{I}=n$ and $\ell_{J}=n+1$. Let $\mathbf{a} \in A^{n}$. Let $\mathbf{b} \in A^{\ell}$ be any tuple such that $b_{i}=a_{i}$ for all $i \in[n], b_{n} \neq b_{n+1}, b_{i_{1}}=b_{i_{2}}$ and $b_{j_{1}}=b_{j_{2}}$. Note that the conditions we placed above for $I$ and $J$ guarantee that such a tuple $\mathbf{b}$ exists. Then there exist tuples $\mathbf{b}^{\prime}, \mathbf{b}^{\prime \prime} \in A^{\ell-1}$ such that $\mathbf{b}=\mathbf{b}^{\prime} \delta_{I}$ and $\mathbf{b}=\mathbf{b}^{\prime \prime} \delta_{J}$, and we have

$$
f\left(a_{1}, \ldots, a_{n-1}, a_{n}\right)=g_{I}\left(\mathbf{b}^{\prime}\right)=g(\mathbf{b})=g_{J}\left(\mathbf{b}^{\prime \prime}\right)=f\left(a_{1}, \ldots, a_{n-1}, b_{n+1}\right) .
$$


Since a was chosen arbitrarily and $a_{n}=b_{n} \neq b_{n+1}$, this implies that the $n$-th argument is inessential in $f$, which contradicts the assumption that ess $f=n$. We conclude that $\ell_{I}=\ell_{J}$ for all $I, J \in\left(\begin{array}{l}\ell \\ 2\end{array}\right)$ with $I, J \nsubseteq E_{L}$.

(Proof of Proposition 9 continued.) By transposing the arguments $n$ and $n+1$ in $g$ if necessary, we may assume that $\ell_{I}=n$ for all $I \in\left(\begin{array}{l}\ell \\ 2\end{array}\right)$ with $I \nsubseteq E_{L}$. Consequently, $g_{I}\left(x_{1}, \ldots, x_{\ell-1}\right)=f\left(x_{1}, \ldots, x_{n}\right)$ for all $I \in\left(\begin{array}{l}\ell \\ 2\end{array}\right)$ with $I \nsubseteq E_{L}$ or $I=L$.

Now, let $\mathbf{u} \in A^{\ell}$. Since $\ell \geq n+|A|$, there exist elements $p, q \in[\ell]$ such that $q \in\{n+1, \ldots, \ell\}$ and $u_{p}=u_{q}$. Let $I=\{p, q\}$. Then $I \nsubseteq E_{L}$ or $I=L$, and there exists $\mathbf{v} \in A^{\ell-1}$ such that $v_{i}=u_{i}$ for all $i \in[n]$ and $\mathbf{u}=\mathbf{v} \delta_{I}$. Therefore, $g(\mathbf{u})=g\left(\mathbf{v} \delta_{I}\right)=g_{I}(\mathbf{v})=f\left(v_{1}, \ldots, v_{n}\right)=f\left(u_{1}, \ldots, u_{n}\right)$. Since $\mathbf{u}$ was chosen arbitrarily, this implies that the last $\ell-n$ arguments of $g$ are inessential, which contradicts the assumption that $g$ depends on all of its arguments.

Let $S \subseteq[n]$, and write

$$
\begin{aligned}
A_{S}^{n}:=\left\{\left(a_{1}, \ldots, a_{n}\right) \in A^{n}: a_{i}\right. & =a_{j} \text { for all } i, j \in[n] \backslash S \text { and } \\
a_{i} & \left.\neq a_{j} \text { for all } i \in S \text { and } j \in[n] \text { with } i \neq j\right\} .
\end{aligned}
$$

Remark 2. If $A$ is finite and $|A|=k$, then $A_{S}^{n} \neq \emptyset$ if and only if $|S| \leq k-1$ or $|S|=k=n$. Furthermore, if $n \geq k+1$ and $S, T \subseteq[n]$ with $|S|=|T| \leq k-1$, then the sets $A_{S}^{n}$ and $A_{T}^{n}$ are disjoint whenever $S \neq T$. Moreover, if $|S| \leq k-1<n$, then $\left|\left\{a_{1}, \ldots, a_{n}\right\}\right|=|S|+1$ for every $\left(a_{1}, \ldots, a_{n}\right) \in A_{S}^{n}$. Note also that if $n=k$, then $A_{S}^{n}=A_{\neq}^{n}$ for every $S$ with $|S|=k-1$.

We say that an $\ell$-element set $S \subseteq[n]$ is stringent in a function $f: A^{n} \rightarrow B$ if the restrictions of $f$ to the sets $A_{S}^{n}$ and $A^{n} \backslash A_{S}^{n}$ do not depend on the arguments indexed by elements of $S$. It clearly holds that if $S=\left\{i_{1}, \ldots, i_{\ell}\right\}$ is stringent in $f$, then there exist functions $h_{\neq}: A_{\neq}^{\ell+1} \rightarrow B$ with Ess $h_{\neq} \subseteq\{1\}$ and $h: A^{n} \rightarrow B$ with Ess $h \subseteq[n] \backslash S$ such that, for all $\left(a_{1}, \ldots, a_{n}\right) \in A^{n}$ and for any $j \in[n] \backslash S$,

$$
f\left(a_{1}, \ldots, a_{n}\right)= \begin{cases}h_{\neq}\left(a_{j}, a_{i_{1}}, \ldots, a_{i_{\ell}}\right), & \text { if }\left(a_{1}, \ldots, a_{n}\right) \in A_{S}^{n}, \\ h\left(a_{1}, \ldots, a_{n}\right), & \text { otherwise. }\end{cases}
$$

Lemma 10. Let $f: A^{n} \rightarrow B$, and assume that $f$ depends on all of its arguments. Then, for every $\ell \in \mathbb{N}_{+}$with $2 \leq \ell<\min (|A|, n-1)$, there exists at most one $\ell$-element subset of $[n]$ that is stringent in $f$.

Proof. Suppose, to the contrary, that $S$ and $T$ are distinct $\ell$-element subsets of $[n]$ that are stringent in $f$. Then there exist elements $s \in S \backslash T$ and $t \in T \backslash S$. We are going to find a contradiction by showing that the $s$-th argument is inessential in $f$. For this goal, let $\mathbf{a}, \mathbf{a}^{\prime} \in A^{n}$ be any tuples satisfying $a_{i}=a_{i}^{\prime}$ for all $i \in[n] \backslash\{s\}$. We want to show that $f(\mathbf{a})=f\left(\mathbf{a}^{\prime}\right)$.

If $\mathbf{a}, \mathbf{a}^{\prime} \in A_{S}^{n}$ or $\mathbf{a}, \mathbf{a}^{\prime} \notin A_{S}^{n}$, then $f(\mathbf{a})=f\left(\mathbf{a}^{\prime}\right)$ by the stringency of $S$. It remains to consider the case when $\mathbf{a} \in A_{S}^{n}$ and $\mathbf{a}^{\prime} \notin A_{S}^{n}$. Since $A_{S}^{n}$ and $A_{T}^{n}$ are disjoint, $\mathbf{a} \notin A_{T}^{n}$. The fact that $\mathbf{a}^{\prime} \notin A_{S}^{n}$ implies that $a_{s}^{\prime}=a_{i}$ for some $i \in[n] \backslash\{s\}$. Hence $\left\{a_{1}^{\prime}, \ldots, a_{n}^{\prime}\right\}=\left\{a_{1}, \ldots, a_{n}\right\} \backslash\left\{a_{s}\right\}$, so $\left|\left\{a_{1}^{\prime}, \ldots, a_{n}^{\prime}\right\}\right|=\left|\left\{a_{1}, \ldots, a_{n}\right\}\right|-1=|S|$. Consequently, $\mathbf{a}^{\prime} \notin A_{W}^{n}$ for all $W \subseteq[n]$ with $|W|=\ell$; in particular, $\mathbf{a}^{\prime} \notin A_{T}^{n}$. Let $\alpha \in\left\{a_{1}, \ldots, a_{n}\right\} \backslash\left\{a_{s}, a_{t}\right\}$ (note that $\left|\left\{a_{1}, \ldots, a_{n}\right\}\right|=|S|+1=\ell+1 \geq 3$ ). Вy changing the $t$-th component of a to $\alpha$ we obtain the tuple $\mathbf{a}_{t}^{\alpha}$. We have $\mathbf{a}_{t}^{\alpha} \notin A_{T}^{n}$, 
because $\ell \leq n-2$, so there exists an element $s^{\prime} \in[n] \backslash(T \cup\{s\})$ and it holds that $a_{s} \neq a_{s^{\prime}}$. By the stringency of $T$, we have

$$
f(\mathbf{a})=f\left(\mathbf{a}_{t}^{\alpha}\right) .
$$

Moreover $\mathbf{a}_{t}^{\alpha} \notin A_{S}^{n}$, because there exists an element $t^{\prime} \in[n] \backslash(S \cup\{t\})$ and the $t$-th and $t^{\prime}$-th components of $\mathbf{a}_{t}^{\alpha}$ are distinct. Changing the $s$-th argument of $\mathbf{a}_{t}^{\alpha}$ to $a_{s}^{\prime}$ we obtain the tuple $\mathbf{b}:=\left(\mathbf{a}_{t}^{\alpha}\right)_{s}^{a_{s}^{\prime}}$ that does not belong to $A_{S}^{n}$ for the same reason as $\mathbf{a}_{t}^{\alpha} \notin A_{S}^{n}: t, t^{\prime} \notin S$ and the $t$-th and $t^{\prime}$-th components of $\mathbf{b}$ are distinct. By the stringency of $S$ we have

$$
f\left(\mathbf{a}_{t}^{\alpha}\right)=f(\mathbf{b}) .
$$

Note that the element $a_{s}$ does not occur in the tuple $\mathbf{b}$, because both $\alpha$ and $a_{s}^{\prime}$ are distinct from $a_{s}$ and there is only one occurrence of $a_{s}$ in $\mathbf{a}$; hence $\left\{b_{1}, \ldots, b_{n}\right\}=$ $\left(\left\{a_{1}, \ldots, a_{n}\right\} \backslash\left\{a_{s}\right\}\right) \cup\left\{a_{s}^{\prime}\right\}$. Now, if $a_{s}^{\prime} \in\left\{a_{1}, \ldots, a_{n}\right\} \backslash\left\{a_{s}\right\}$, then $\left|\left\{b_{1}, \ldots, b_{n}\right\}\right|=\ell$, whence $\mathbf{b} \notin A_{T}^{n}$. If $a_{s}^{\prime} \notin\left\{a_{1}, \ldots, a_{n}\right\} \backslash\left\{a_{s}\right\}$, then $b_{s}=a_{s}^{\prime} \neq a_{s^{\prime}}=b_{s^{\prime}}$, with $s, s^{\prime} \notin T$, so $\mathbf{b} \notin A_{T}^{n}$. By changing the $t$-th component of $\mathbf{b}$ to $a_{t}$ we get the tuple $\mathbf{a}^{\prime}$. We have observed above that $\mathbf{a}^{\prime}$ is not in any set of the form $A_{W}^{n}$, in particular, $\mathbf{a}^{\prime} \notin A_{T}^{n}$. It follows from the stringency of $T$ that

$$
f(\mathbf{b})=f\left(\mathbf{a}^{\prime}\right) .
$$

Putting (2), (3), and (4) together, we see that the equality $f(\mathbf{a})=f\left(\mathbf{a}^{\prime}\right)$ holds also in this case.

Since the choice of $\mathbf{a}$ and $\mathbf{a}^{\prime}$ above was arbitrary, this shows that the $s$-th argument is inessential in $f$. This contradicts the assumption that $f$ depends on all of its arguments. We conclude that there is at most one $\ell$-element subset of $[n]$ that is stringent in $f$.

Proposition 11. Let $f: A^{n} \rightarrow B$, and assume that $f$ depends on all of its arguments. Then for every $\ell \in \mathbb{N}_{+}$with $1 \leq \ell<|A|$, there exists an upper cover of $f$ with $n+\ell$ essential arguments.

Proof. The claim is obvious if $\ell=1$, so let us assume that $\ell \geq 2$ and hence $|A| \geq 3$. In the following, $\phi: A_{\neq}^{\ell+1} \rightarrow B$ is a map that will be specified later. Define $g: A^{n+\ell} \rightarrow B$ by the rule

$$
g\left(x_{1}, \ldots, x_{n+\ell}\right)= \begin{cases}\phi\left(x_{n}, \ldots, x_{n+\ell}\right), & \text { if } x_{1}=\cdots=x_{n} \text { and } \\ & x_{n}, \ldots, x_{n+\ell} \text { are distinct } \\ f\left(x_{1}, \ldots, x_{n}\right), & \text { otherwise }\end{cases}
$$

The function $\phi$ must be chosen in such a way that the function $g$ depends on all of its arguments. This can be achieved, for example, by letting $\phi: A_{\neq}^{\ell+1} \rightarrow B$ be the function $\phi\left(x_{1}, \ldots, x_{\ell+1}\right)=\gamma\left(x_{1}\right)$, where $\gamma: A \rightarrow B$ is an arbitrary unary map distinct from $\Delta f$. (It is straightforward to verify that the resulting function $g$ depends on all of its arguments.) We would also like to make sure that $g$ is an upper cover of $f$, so a bit more care must be taken, and the exact choice of $\phi$ depends on $f$ as will be discussed below.

Let us consider the identification minors of $g$. Let $I=\{i, j\} \in\left(\begin{array}{c}n+\ell \\ 2\end{array}\right)$ with $i<j$. If $j \geq n+1$, then, by the definition of $g$, we have

$$
g_{I}\left(x_{1}, \ldots, x_{n+\ell-1}\right)=f\left(x_{1}, \ldots, x_{n}\right),
$$


so $g$ is a major of $f$, as desired. If $j \leq n$, then

$$
g_{I}\left(x_{1}, \ldots, x_{n+\ell-1}\right)= \begin{cases}\phi\left(x_{n-1}, \ldots, x_{n+\ell-1}\right), & \text { if } x_{1}=\cdots=x_{n-1} \text { and } \\ & x_{n-1}, \ldots, x_{n+\ell-1} \text { are distinct } \\ f_{I}\left(x_{1}, \ldots, x_{n-1}\right), & \text { otherwise }\end{cases}
$$

In this case, it might happen that $f<g_{I}$. We want to avoid this by choosing $\phi$ carefully. Note first that if we identify one of the last $\ell$ arguments with any other argument in $g_{I}$, we get $f_{I}$, which is fine. We might still be able to get $f$ when we identify some arguments among the first $n-1$.

In order to analyse the situation, let $\Pi$ be an $n$-partition of $[n+\ell]$ such that $\{n+1\},\{n+2\}, \ldots,\{n+\ell\} \in \Pi$, and let $\Pi^{\prime}$ be the $(n-\ell)$-partition of $[n]$ whose blocks are the $\Pi$-blocks included in $[n]$. If $\ell \geq n$, then no such partition $\Pi$ exists and we are done: we may take $\phi\left(x_{1}, \ldots, x_{\ell+1}\right)=\gamma\left(x_{1}\right)$, where $\gamma: A \rightarrow B$ is any map distinct from $\Delta f$. Assume that $\ell \leq n-1$. Then

$$
g_{\Pi}\left(x_{1}, \ldots, x_{n}\right)= \begin{cases}\phi\left(x_{n-\ell}, \ldots, x_{n}\right), & \text { if } x_{1}=\cdots=x_{n-\ell} \text { and } \\ & x_{n-\ell}, \ldots, x_{n} \text { are distinct } \\ f_{\Pi^{\prime}}\left(x_{1}, \ldots, x_{n-\ell}\right), & \text { otherwise. }\end{cases}
$$

If $\ell=n-1$, then the above expression of $g_{\Pi}$ simplifies to

$$
g_{\Pi}\left(x_{1}, \ldots, x_{n}\right)= \begin{cases}\phi\left(x_{1}, \ldots, x_{n}\right), & \text { if }\left(x_{1}, \ldots, x_{n}\right) \in A_{\neq}^{n}, \\ \Delta f\left(x_{1}\right), & \text { otherwise. }\end{cases}
$$

Let $\gamma: A \rightarrow B$ be a unary map distinct from $\Delta f$ such that $\gamma$ is constant if and only if the restriction $\left.f\right|_{A_{\neq}^{n}}$ is not constant (it is clear that such a map $\gamma$ exists). Let $\phi: A_{\neq}^{n} \rightarrow B$ be the map $\phi\left(x_{1}, \ldots, x_{n}\right)=\gamma\left(x_{1}\right)$. Then $g$ is a major of $f$ with $n+\ell$ essential arguments and $g_{\Pi} \not \equiv f$.

Finally, consider the case when $\ell \leq n-2$. Let $\gamma: A \rightarrow B$ be an arbitrary unary map distinct from $\Delta f$. If $f$ has a stringent set $S$ of cardinality $\ell$ (by Lemma 10 such a set $S$ is unique if one exists) and a representation as in equation (1) with $h_{\neq}: A_{\neq}^{\ell+1} \rightarrow B$ and $h: A^{n} \rightarrow B$, then we additionally require that $\gamma$ is constant if and only if $h_{\neq}$is not constant. It is clear that such a function $\gamma$ exists. Let $\phi: A_{\neq}^{\ell+1} \rightarrow B$ be the map $\phi\left(x_{1}, \ldots, x_{\ell+1}\right)=\gamma\left(x_{1}\right)$. Then $g$ is a major of $f$ with $n+\ell$ essential arguments. Since Ess $\phi \subseteq\{1\}$, the set $T:=\{n-\ell+1, \ldots, n\}$ is stringent in $g_{\Pi}$. It is not difficult to see that $g_{\Pi} \not \equiv f$, either because $g_{\Pi}$ has a stringent set of cardinality $\ell$ but $f$ does not, or because the restriction of $g_{\Pi}$ to the set $A_{T}^{n}$ is not equivalent to the restriction of $f$ to $A_{S}^{n}$.

We conclude that $g$ is an upper cover of $f$, as desired.

Proof of Theorem 5. Follows immediately by putting together Propositions 8, 9, and 11.

\section{Concluding Remarks}

In Theorem 5 we determined for every function $f: A^{n} \rightarrow B$ the maximum essential arity of the upper covers of $f$. In fact, we showed that for each $1 \leq \ell<|A|$ there exists an upper cover of essential arity $n+\ell$. This gives lower bounds on the number of upper covers that a function may have. In the case when $A$ is infinite, every function has infinitely many upper covers, whereas if $A$ is finite, then there 
are at least $|A|-1$ upper covers. In the latter case, determining the exact number remains an open problem that is a topic of further research.

Question 4. Estimate $\left|C_{f}\right|$.

\section{ACKNOWLEDGMENTS}

The authors wish to thank the anonymous referees for most valuable comments, suggestions, and questions, which helped improve the presentation and sharpen the current results.

\section{REFERENCES}

[1] Berman, J., Kisielewicz, A.: On the number of operations in a clone. Proc. Amer. Math. Soc. 122, 359-369 (1994)

[2] Bouaziz, M., Couceiro, M., Pouzet, M.: Join-irreducible Boolean functions. Order 27, 261-282 (2010)

[3] Couceiro, M., Lehtonen, E.: Generalizations of Świerczkowski's lemma and the arity gap of finite functions. Discrete Math. 309, 5905-5912 (2009)

[4] Couceiro, M., Lehtonen, E., Waldhauser, T.: Parametrized arity gap. Order 30, 557-572 (2013)

[5] Couceiro, M., Pouzet, M.: On a quasi-ordering on Boolean functions. Theoret. Comput. Sci. 396, 71-87 (2008)

[6] Lehtonen, E., Szendrei, Á.: Partial orders induced by quasilinear clones. Contributions to General Algebra 20, Proceedings of the Salzburg Conference 2011 (AAA81), Verlag Johannes Heyn, Klagenfurt, pp. 51-84 (2012)

[7] Willard, R.: Essential arities of term operations in finite algebras. Discrete Math. 149, 239-259 (1996)

(M. Couceiro) LORIA (CNRS - Inria Nancy Grand Est - Universit de Lorraine), CamPus scientifique, BP 239, 54506 Vandeuvre-LS-Nancy Cedex, France

E-mail address: miguel.couceiro@inria.fr

(E. Lehtonen) Technische Universität Dresden,, Institut für Algebra, 01062 Dresden, Germany

E-mail address: Erkko.Lehtonen@tu-dresden.de 